\title{
ACTIVITY/POSTURE RECOGNITION USING WEARABLE SENSORS PLACED ON DIFFERENT BODY LOCATIONS
}

\author{
Hristijan Gjoreski, Matjaž Gams \\ Department of Intelligent Systems \\ Jožef Stefan Institute \\ Jamova cesta 39, 1000 Ljubljana, Slovenia \\ E-mail: \{hristijan.gjoreski, matjaz.gams\}@ijs.si
}

\begin{abstract}
This paper presents an approach to activity/posture recognition using wearable sensors. The sensors consist of 3-axis accelerometer, 3D gyroscope and 3D magnetometer (3D compass). Several approaches of activity and posture recognition are analyzed, varying the number, type of the sensors and using different body placements. The effect of these different scenarios on the accuracy is investigated. Placements of up to three sensors are considered: chest, right thigh and right ankle. Extraction of additional attributes was performed in order to more precisely reconstruct the activities/postures. The results indicate that the gyroscope and magnetometer data significantly improve performance of the system, in particular when only one sensor was placed on the chest.
\end{abstract}

\section{KEY WORDS}

Artificial intelligence, intelligent systems, data mining, ambient intelligence, activity recognition, posture recognition, wearable sensors.

\section{Introduction}

There are a lot of projects and useful applications aiming at nursing older people. Most of them belong to the area of ambient intelligence and are trying to make an everyday life easier, simpler and safer for elderly. Improving the quality of life of Europe's increasingly elderly population is one of the most pressing challenges facing our society today. Nearly $14 \%$ of the EU population is over the age of 65 and this figure is expected to double by 2050 [1]. By then Europe will have 80 million older citizens who should continue to play an active role in our society, despite limitations which the ageing process often brings. To cope with this situation, intelligent systems and techniques are being developed, which can give these people self-confidence to actively and independently live their life despite their age limitations.

The activity/posture is an important task in ambient assisted living (AAL). In this paper we present our work on the activity/posture recognition using wearable sensors. Each sensor consist three sensor modules: accelerometer, gyroscope (gyro) and magnetometer. After analyzing the related studies for this task in terms of the sensors that are used, we noticed that most of the studies use only accelerometers or in some studies combinations with gyroscopes. Therefore we decided to make a comparison using only the acceleration module on one side and all three modules on the other side.

In activity/posture recognition process we focused on seven basic body activities/postures. We also investigated the performances with different numbers of sensors (1 to 3 ) and different placements on the body (chest, ankle and thigh). This way we showed the trade-off between the intrusiveness of the system and the achieved accuracy. The final system should be as non-intrusive as possible (fewer wearable sensors), but still accurate enough to detect each activity/posture.

The classification algorithm used for the research is Random Forest. This was the algorithm yielding the best results after analyzing several classification algorithms. For the experiments 11 young people were recorded performing the same scenario 5 times each. The leaveone-person-out (i.e. cross validation on 11 people) technique was used for evaluating the results.

\section{Related Work}

In most of the studies the researchers are using accelerometers or a combination of accelerometers and gyroscopes (gyros). There are also some studies that use location systems. However, each of these approaches has certain problems.

The location system approach is not commonly used, but some reported results are in [2, 3, 4, 5]. In [2] they used 4 location tags fixed to the user's body and the achieved accuracy is around $87 \%$ for the machine learning approach. The evaluation approach is: training the classifier on 2 persons and testing on third person. They also tested another approach using common rules and the achieved accuracy is $89 \%$. The problem with the location systems is the variation of the sensors precision (not constant rate of distance error) and price of the sensors. For reasonable results high precision is required 
[6], resulting in high cost of sensors. This price is many times higher than the price of accelerometers or gyros. In addition, accelerometers and gyros can be found almost in any smart phone. The localization systems are usually limited to indoor environment and require installation in each room. The advantage of location sensors is automatic detection of person's location.

Most of the researchers investigate the activity/posture recognition problem using accelerometers. Accelerometers have been widely accepted as useful and practical sensors for wearable devices to measure and assess physical activity/posture. The most common approach is data mining [7, 8, 9]. Of particular interest are the results presented in [7]. When they use the same person for training and testing the achieved accuracy was $90 \%$, but when they use different person for testing the accuracy was $65 \%$. In our research we use different people for training and testing. Similar evaluation approach to ours is used in [9]. Leave-oneperson-out cross validation technique is performed. They used the data from 6 people for training and one person for testing. They used Neuro-Fuzzy classifier and one accelerometer fixed on the wrist. Some of the target activities/postures differ from ours. The presented results show $84 \%$ accuracy.

There are studies that use manually created algorithms [10]. With this approach the achieved accuracy is high for specific scenarios. But this approach requires multiple number of accelerometers (in [10] they used 6). Our assumption is that for reasonable results we need only few wearable sensors, therefore the system would be simple, user friendly and low cost.

Several studies on activity/posture recognition use accelerometers and gyroscopes. In [11] they used one 2axis accelerometer and gyroscopes worn on the chest; by applying thresholds to accelerations, angular velocities and angles, they detected a potential fall and the activity/posture after the fall. The reported overall accuracy is $90 \%$, but their evaluation approach is different. In our research we try to predict each data sample not averaging over longer time periods, e.g. lying for 20 seconds as one sample. Similar threshold algorithm is used in [12], but their focus is more on detection of falls. The hardware is consisted of two 3-axis accelerometers and gyroscopes, worn on the chest and thigh.

\section{Sensors}

The sensors used in this research are three wearable Xsens-MTx sensors [13]. Each sensor is a complete miniature inertial measurement unit equipped with: 3 -axis accelerometer, 3D gyroscope and 3D magnetometer. Moreover it has an embedded processor capable of extraction of the orientation and rotation in real time, as well as outputting calibrated 3D linear acceleration, rate of turn (gyroscope) and (earth) magnetic field data.

A 3-axis accelerometer is a sensor that returns a realvalued estimate of the acceleration along the $\mathrm{x}, \mathrm{y}$ and $\mathrm{z}$ axes from which velocity and orientation angle can also be estimated. Accelerometers measure the acceleration and output the projections of the acceleration vector represented in a three dimensional coordinate system. Each accelerometer has its own coordinate system and gives the relative vector projections. Because of the Earth's gravity, all objects experience a gravitational pull towards the Earth's centre. The acceleration unit of the pull is referred to as $\mathrm{g}$ or $\mathrm{g}$ force. Consequently all objects are subject to $1 \mathrm{~g}$ acceleration (Figure 1). When the accelerometer is at rest, only Earth's gravity is measured. Accelerometers can be used as motion detectors as well as for body-posture recognition and fall detection.

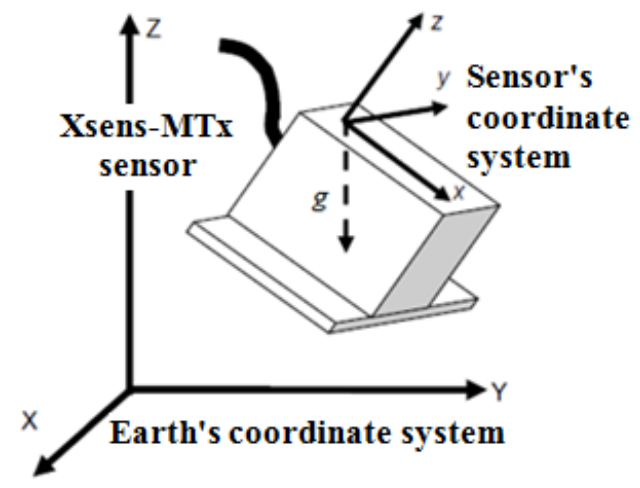

Figure 1: Earth-specific and sensor-specific 3D coordinate systems, and the Earth's gravity - g.

A gyroscope or gyro is a device for measuring or maintaining orientation, based on the principles of conservation of angular momentum. It will keep the original direction of its rotation axis no matter which way the sensor is turned. The data received from this module is useful in activity/posture recognition process because of the estimation of the sensor orientation and rotation. The advantage compared to accelerometers orientation estimation is in dynamic activities/postures. During these activities/postures the user individual acceleration affects the estimation of the orientation using only acceleration data, which corrupts the final estimation. In these situations the gyroscope and magnetometer data are used as addition in orientation estimation. In general, gyroscope is being used in compasses, aircraft, computer pointing devices, etc. In recent years, they have been introduced into consumer electronics. Since the gyroscope allows the calculation of orientation and rotation, designers have incorporated them into modern technology. The integration of the gyroscope has allowed more accurate recognition of movement within a 3D space than the previous single accelerometer within a number of smart phones. 
The heading of the sensor is stabilized using the magnetometer, which measures the local (earth) magnetic field. In other words, the measured magnetic field is used as a compass. If the local Earth magnetic field is temporarily disturbed, the sensor will track this disturbance instead of incorrectly assuming there is no disturbance. However, in case of structural magnetic disturbance $(>10$ to $20 \mathrm{~s}$ ) the computed heading will slowly converge to a solution using the 'new' local magnetic north. Note that the magnetic field has no direct effect on the inclination estimate.

The sensors used in this research have data sampling frequency of $6 \mathrm{~Hz}$. This is not a high frequency, but it gives enough information for our final goal and makes the system more compact and portable even on devices with low memory and low processing power. It is also equal to the sampling frequency of the location sensors we used for comparison.

\section{System Architecture}

The activity/posture recognition process is divided in several phases (Figure 2). It begins with the real world in which wearable sensors are affixed to a person's body. The sensors sample the signal and send the raw data to the software attribute extraction module. The software analyzes the raw data and extracts new attributes. To solve the problem of activity/posture recognition, a classification module is developed. This module gives an output: the real time activity/posture of the body.

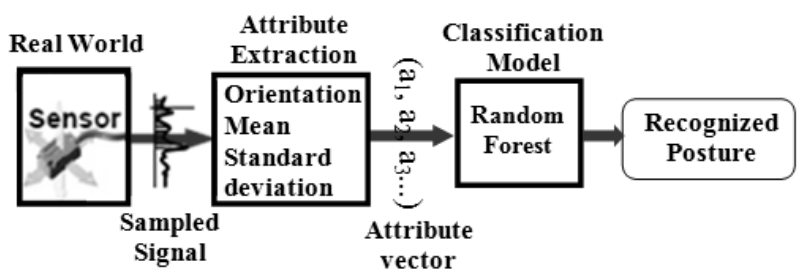

Figure 2: System Architecture

The data mining module classifies the sensor data samples into 7 predefined activities/postures: standing, sitting, lying, on all fours, sitting on the ground standing up and going down (which includes sitting down, lying down or falling). The process of walking is included into posture standing, therefore standing can be a static or a dynamic activity/posture.

Each sensor experiences the gravitational acceleration. This is an important reference for calculating sensor orientation angle and distinguishing different body activities/postures. The orientation angle using only acceleration data is extracted as the angle between the acceleration vector ( $\mathrm{g}-$ if the posture is static) and each of the axis unit vectors ( $\mathrm{x}, \mathrm{y}$ and $\mathrm{z}$ ). When the data from gyro and magnetometer was included, the processor and software module in the sensor itself were able to calculate the orientation - quaternions, which gave better orientation estimate than the acceleration estimate.

Using this orientation attributes the system has a unique sensor space orientation. With the orientation angle attributes, the data mining algorithm can easily distinguish static postures that have different sensor orientation angles. For instance the chest sensor is perpendicular to the ground for standing and sitting postures, but parallel to the ground for lying and on all fours. Because of such similarities and differences in the orientation of the sensors the body postures can be recognized. Also a special movement attribute is extracted for distinguishing dynamic from static activities/postures. This attribute captures the changes in the acceleration vector; the greater the changes, the fiercer the motion.

The number and placements of the sensors on the body can affect the recognition of particular activities/postures. Placing a sensor on the thigh can help distinguishing sitting (sensor is parallel to the ground) and standing (sensor is perpendicular to the ground), but distinguishing sitting and lying is a problem. On the other hand, a sensor on the chest can distinguish sitting and lying, but has problems with standing and sitting. By combining these two sensors the algorithm was able to distinguish each of the discussed activities/postures. Because of situations like this, we decided to compare the results using different numbers of sensors and different body placements. The idea is to use as few sensors as possible to maximize the user's comfort, but to use enough of them to achieve satisfactory performance. It is very important that the sensors are firmly fixed, because the orientation of the sensor is the most important feature. The body placements of the sensors were chosen to be: chest, right thigh and the right ankle.

\section{Data Preparation}

In this section we describe the methods and algorithms used in our system. We used supervised classification techniques; therefore the data had to be manually labelled with the appropriate class (activity/posture). Because the labelling was done online, small delays in labels can happen. Therefore, successive activities (e.g. standing -> going down -> lying) may have some samples mislabelled particularly at the beginning and at the end of each activity/posture. This can affect the recognition accuracy especially for short activities/postures (going down or standing up) that do not have lots of samples. In addition, the border between two successive activities/postures is to some degree subject to interpretation.

After the process of attribute extraction (which is explained in the next 2 subsections) all attributes from each sensor are collected together in one attribute vector. This vector is passed through to the classification model, which tries to predict the appropriate activity/posture. The 
classification model was previously trained. The decision which classification algorithm to be used was made after evaluating the results in the Weka toolkit. Several commonly used classification algorithms were analyzed: SVM, J48 and Random Forest. The algorithm that achieved the best results was Random Forest.

Random Forest is supervised machine learning algorithm [14]. It is an ensemble classifier that consists of many individual decision trees (in our case 10). We decided to use this algorithm after the preliminary results and also because it is designed to operate quickly over large datasets and more importantly to be diverse by using random samples to build each tree in the forest.

\subsection{Accelerometer based Activity/Posture Recognition}

The first approach that we will discuss is the accelerometers based activity/posture recognition. In this approach we used only the data from the acceleration module. The first set of attributes are the raw acceleration vector projections ( 3 attributes for each accelerometer) $a_{\mathrm{x}}$, $a_{\mathrm{y}}$ and $a_{\mathrm{z}}$. Additional 18 attributes are extracted for each sensor: length of the acceleration vector (1 attribute), orientation angles for each axis $-\mathrm{x}, \mathrm{y}$ and $\mathrm{z}$ (3 attributes), statistical attributes for each axis and for the length of the acceleration vector (mean value (4 attributes), root mean square (4 attributes), standard deviation (4 attributes)), movement detection attributes (2 attributes).

The first derived attribute is the length (module) of the acceleration vector. It is a simple but very useful attribute, which is also used further in the process of extraction of new attributes. Its definition is:

$$
l=\sqrt{a_{\mathrm{x}}^{2}+a_{\mathrm{y}}^{2}+a_{\mathrm{z}}^{2}} .
$$

During static postures this attribute is constant with the value equal to the Earth's gravity $(l=1 g)$. In dynamic activities the acceleration vector is changing the direction and its length.

Important characteristics for static body posture recognition are the orientation angles of the accelerometer. The orientation angles are calculated as the angles between the actual acceleration (Earth's gravity for static activities/postures) and each of the axes (x, y and $\mathrm{z}$ ). For instance, the angle $\varphi_{x}$ between the acceleration vector and the $\mathrm{x}$ axis (perpendicular to the ground) is computed as follows:

$$
\cos \varphi_{x}=\frac{a_{x}}{\sqrt{a_{\mathrm{x}}^{2}+a_{\mathrm{y}}^{2}+a_{\mathrm{z}}^{2}}} .
$$

This attribute improves the classification for static activities/postures that have different sensor angle orientations. Since each person has his/her characteristic posture and each accelerometer may not always be worn in exactly the same way, a method for the adaptation to the user is performed. At the beginning of each recording for each person there is initialization (normalization) period of 15 seconds. The average orientation angle of standing was measured as $\varphi_{0}$. The difference between the "ideal" standing orientation angle (e.g. $180^{\circ}$ for the $\mathrm{x}$ axis) and $\varphi_{0}$ was calculated as $\varphi_{\text {diff }}=\varphi_{\text {ideal }}-\varphi_{0}$. After the normalization period, to each newly calculated orientation angle $\varphi_{i}$ the difference angle $\varphi_{\text {diff }}$ is added and finally the normalized angle is calculated as $\varphi_{\text {normal }}=\varphi_{i}+\varphi_{\text {diff. }}$. This adaptation procedure is performed for each axis for each accelerometer. Without this adaptation technique the model and the results were person dependable. There was a big difference in the accuracy for people wearing the accelerometers in slightly different way.

A sliding window is used for calculation of the statistical attributes. The current data sample and 5 past data samples are combined in one window. The window size is chosen to be 6 data samples ( 1 second interval) because in this task there are short-lasting activities that the system should detect (going down, standing up). The first attribute from this group is the Mean value of the data in the window. This attribute is actually performing a low-pass filter to the raw data. The filtered data is smoother and has fewer changes. This is a good feature for the activity/posture recognition process. The mathematical definition for the $\mathrm{x}$ axis is:

$$
\operatorname{Mean}_{x}=\frac{\sum_{i=1}^{n} a_{x}^{i}}{n} .
$$

The number of data samples $n$ is 6 ( 1 second window size). The variable $a_{x}^{i}$ is the acceleration along $\mathrm{x}$ axis. Using the same formula, mean values for other axes $M e a n_{\mathrm{y}}$ and $M e a n_{\mathrm{z}}$ are calculated. Also the mean value for the length of the acceleration vector Mean ${ }_{\text {length }}$ is calculated. A similar approach is used for the Standard deviation and the Root mean square attributes.

The Root Mean Square is a similar attribute to the Mean value, but it is useful when the observed value is varying above and below zero. That is the case in our acceleration values. Depending of the orientation of the accelerometer, the values can be positive or negative (e.g. $+\mathrm{g}$ or $-\mathrm{g}$ ). The RMS for the length of the acceleration vector is computed as follows:

$$
R M S_{\text {length }}=\sqrt{\frac{\sum_{i=1}^{n} l_{i}^{2}}{n}} .
$$

The variable $l_{i}^{2}$ is the square of the length of the acceleration vector for the current member in the sum. Similarly the $R M S_{\mathrm{x}}, R M S_{\mathrm{y}}$ and $R M S_{\mathrm{z}}$ were calculated.

The Standard Deviation attribute is good for distinguishing long-lasting static activities/postures from transitional ones. It can detect when the movement of the accelerometer is intense. The mathematical definition for the length of the acceleration vector is:

$$
S T D_{\text {length }}=\sqrt{\frac{\sum_{i=1}^{n}\left(l_{i}-\bar{l}\right)^{2}}{n}} .
$$

The variable $l_{i}$ is the length for the current member in the sum and $\bar{l}$ is the mean value in the current window. Also the standard deviation for each of the axes was calculated: $S T D_{\mathrm{x}}, S T D_{\mathrm{y}}$ and $S T D_{\mathrm{z}}$. 
When a person's body is static, the accelerometers respond only to the gravity, producing a constant $1 g$ total acceleration. During motion the accelerometers produce changing acceleration signal and the fiercer the motion, the greater the change in the signal. Using these changes in the acceleration vector an attribute is computed for the detection of sensor movement - Acceleration Changes $(A C)$. AC value of this attribute increases as the accelerometer is in movement (walking, going down, standing up etc.). This attribute takes in consideration the last 2 seconds of data (12 data samples). It sums up the last 12 differences of lengths of the acceleration vector and divides the sum with the time interval ( 2 seconds) of the data. AC is computed as follows:

$$
A C=\frac{\sum_{i=0}^{n-1}\left|l_{i+1}-l_{i}\right|}{T_{n}-T_{0}} .
$$

$T_{0}$ is the time stamp for the first data sample in the window and $T_{n}$ is the time stamp of the last data sample. With this attribute the movement of the person can be detected: it distinguishes static from dynamic activities/postures. A boolean (true/false) attribute, which compares the AC attribute value to a threshold, is also computed. If the value is above the threshold, the boolean attribute is true, otherwise it is false. The threshold value is 0.0015 and it was chosen empirically after series of tests on recordings different from the test ones.

All these 21 attributes were extracted for each accelerometer module and combined together in one attribute vector. Afterwards, the classification model predicts the appropriate activity/posture. The achieved results are described in the next section.

\subsection{Accelerometer, Gyroscope and Magnetometer Activity/Posture Recognition}

In this approach we used the data from the three sensor modules placed in the Xsens-MTx sensor. From the acceleration module we used the attributes that are explained in the previous subsection. The only difference is that we excluded the orientation angles attributes. The reason for this is the more accurate orientation estimation that is done by combining the data from the three sensor modules. The Xsens-MTx system has its own hardware and software processing unit that is capable of computing additional orientation attributes besides the raw data. This unit uses signals of the gyroscopes, accelerometers and magnetometers to compute a statistical optimal 3D orientation estimate of high accuracy with no drift for both static and dynamic movements. This new, better orientation can be expressed by quaternions or Euler angles.

A quaternion is a four-dimensional construct loosely defined as an axis and a rotational component around the defined axis (usually represented as $\{\mathrm{x}, \mathrm{y}, \mathrm{z}, \mathrm{w}\}$ ). Unit quaternions provide a convenient mathematical notation for representing orientations and rotations of objects in three dimensions. They are simple and efficient to interpolate and represent a single orientation unambiguously, which is not the case with Euler angles.

The other representation of the rotation and orientation of the sensor is by using the Euler angles (Roll, Pitch and Yaw/Heading). This representation is more suitable for human understanding (Figure 3), but has several disadvantages. Compared to quaternions, Euler angles have the problem of singularity. So, one must be aware of singularities in the Euler angle parameterization when the pitch approaches $\pm 90^{\circ}$ (north/south pole). These cases must be handled specially. Because the system was able to provide only one information at a time (quaternions or Euler angles), we decided to use the unambiguous representation - quaternions as "raw" data from the Xsens system. The conversion from quaternions to Euler angles is mathematically defined in [15]. Using those equations we extracted the Euler angles afterwards in our attribute extraction module. Therefore, in the final attribute vector we included the attributes from both representations.

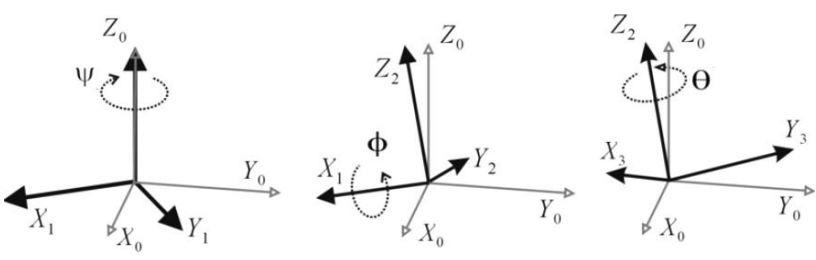

Figure 3: A rotation represented by Euler angles (pitch, roll yaw) with $(\psi, \varphi, \theta)=,\left(-60^{\circ}, 30^{\circ}, 45^{\circ}\right)$.

By default, the heading of the sensor is stabilized using the magnetometer data - the local (earth) magnetic field. The measured magnetic field is used as a compass. This information from the magnetometer is included in the process of extracting the quaternions. No additional data from the magnetometer is analyzed and used.

Beside these new orientation attributes (seven) we included three more attributes from the raw data from the gyroscope - along the 3 axes (x, y and $\mathrm{z}$ ). These attributes actually express the angular velocity vector in 3D coordinate system. This is similar to the acceleration raw attributes, but in gyroscope data the measured vector is the angular velocity instead of acceleration.

With these attributes the construction of the attribute vector is concluded. In this approach it was consisted of 28 attributes (10 new and 18 from the acceleration data) for each sensor.

\section{Experimental Results}

We compared the performances of the activity/posture recognition approaches on the 14 minutes long testing scenario. It includes all previously discussed target activities/postures. It was recorded by 11 young healthy volunteers 7 male and 4 female. The events are listed in Table 1. They were recorded in a single recording 
interspersed with short periods of walking. The scenario was recorded in a laboratory. Each person repeated the scenario 5 times. That resulted in 55 recordings and overall around 280000 data samples (6 data samples per second).

Table 1: Events sequence in the test scenario.

\begin{tabular}{l|l}
\hline No. & Description \\
\hline 1 & Sitting down on the chair and sitting. \\
2 & Lying down quickly on the ground and lying. \\
3 & Lying down on the bed and lying. \\
4 & Lying down slowly on the ground and lying. \\
5 & $\begin{array}{l}\text { Sitting down quickly on the chair and sitting. } \\
6\end{array}$ \\
7 & $\begin{array}{l}\text { Lying down quickly on the bed and lying. } \\
\text { ground down from chair and sitting of the } \\
8\end{array}$ \\
\hline
\end{tabular}

The distribution of the data samples (instances) over class attribute is not uniform. Two activities are very quick and last for a couple of seconds (standing up, going down). Therefore it was not possible to have equal number of instances for these activities. But for the other 5 target activities/postures the number of instances is almost equal.

The leave-one-person-out technique was used for evaluating the results. That means the model was trained on 10 people and tested on the remaining person. This procedure was repeated for each person (11 times). At the end the average accuracy was calculated for each activity/posture separately and the overall accuracy was also calculated. This evaluation approach is more reliable than the ones that use the same person for training and testing. Using the same person would give overly optimistic results if the intended use of the model is to classify the activities/postures of previously unseen people.

Because there is a lot of related work that presents accuracies using different numbers and placements of sensors, we included four different sensor combinations in our table. The results are shown in Table 2. It should be noted that sensors placed only on the thigh or ankle did not achieve reasonable results, so they are omitted from the final table. Actually the results showed that the chest sensor placement is the best suited for activity/posture recognition task.

In a system with one sensor placed on the chest the results are as expected:

- It is difficult to distinguish sitting and standing, or sitting and sitting on the ground. This is because the orientation of the sensor is similar during these static activities/postures.

- On all fours is usually confused with lying on the stomach, because the sensor orientation is the same (parallel to the ground and facing the ground).

- Transitional activities (standing up and going down) are difficult to distinguish and are usually mutually misclassified or confused with dynamic standing walking. Also there are small errors with other activities/postures because of the mistakes in manual data labeling.

When we included the gyroscope and magnetometer data the overall accuracy improved by 17 percentage points. This is because of the improved estimation of sensor orientation and rotation using the quaternions and Euler angles.

Another thing that is obvious is the improvement in accuracy when the system is using two sensors. When the chest sensor is combined with the right ankle sensor, there is a noticeable improvement in sitting and sitting on the ground. That is the case in both sensor scenarios. The reason for this improvement is different orientation of the ankle during sitting on a ground and sitting on a chair. When chest sensor is combined with the right thigh sensor, there is an improvement in distinguishing between sitting and standing, and also lying and on all fours. The orientation of the thigh sensor in both cases is parallel vs. perpendicular to the ground.

With introducing the third sensor in the system there is an improvement in each of the activities/postures. Actually it is a combination of the achieved accuracies using two sensors on different locations. It should be emphasized that $100 \%$ accuracy is almost impossible because of the previously (in section IV) discussed issue with the labelling.

If we compare the results in the different sensor scenarios, we can see that the gyroscope and magnetometer data significantly improve the accuracy of the system in prediction of each of the 7 target activities/postures. The most significant overall improvement (almost 15 percentage points) is noticed while the system is using one sensor placed on the chest. 
Table 2: Comparison of activity/posture recognition accuracy using different number of sensors $(1,2$ or 3$)$ placed on the Chest, Ankle Right (AR) and Thigh Right (TR).

\begin{tabular}{|c|c|c|c|c|c|c|c|c|}
\hline Random Forest & \multicolumn{4}{|c|}{ Accelerometer } & \multicolumn{4}{|c|}{ Accelerometer + Gyroscope + Magnetometer } \\
\hline $\begin{array}{l}\text { Activity/ } \\
\text { Posture }\end{array}$ & Chest & $\begin{array}{c}\text { Chest + } \\
\text { AR }\end{array}$ & $\begin{array}{c}\text { Chest + } \\
\text { TR }\end{array}$ & $\begin{array}{c}\text { Chest + } \\
\text { AR + TR }\end{array}$ & Chest & $\begin{array}{c}\text { Chest + } \\
\text { AR }\end{array}$ & $\begin{array}{c}\text { Chest + } \\
\text { TR }\end{array}$ & $\begin{array}{c}\text { Chest + } \\
\text { AR + TR }\end{array}$ \\
\hline Lying & $94.9 \%$ & $96.0 \%$ & $95.0 \%$ & $98.0 \%$ & $97.7 \%$ & $97.8 \%$ & $98.5 \%$ & $99.2 \%$ \\
\hline Sitting & $40.2 \%$ & $70.5 \%$ & $\mathbf{7 8 . 0 \%}$ & $89.2 \%$ & $82.4 \%$ & $80.6 \%$ & $93.4 \%$ & $98.0 \%$ \\
\hline Standing & $\mathbf{7 7 . 5 \%}$ & $88.9 \%$ & $93.9 \%$ & $97.4 \%$ & $87.8 \%$ & $95.3 \%$ & $98.7 \%$ & $99.1 \%$ \\
\hline On all fours & $48.9 \%$ & $\mathbf{5 8 . 8 \%}$ & $\mathbf{7 4 . 3 \%}$ & $88.3 \%$ & $\mathbf{5 2 . 8 \%}$ & $\mathbf{5 9 . 3 \%}$ & $83.7 \%$ & $92.5 \%$ \\
\hline Sit on ground & $45.7 \%$ & $88.3 \%$ & $85.9 \%$ & $89.3 \%$ & $\mathbf{5 9 . 9 \%}$ & $90.4 \%$ & $86.3 \%$ & $92.4 \%$ \\
\hline Going down & $36.1 \%$ & $43.3 \%$ & $45.8 \%$ & $53.0 \%$ & $44.5 \%$ & $46.2 \%$ & $55.1 \%$ & $63.3 \%$ \\
\hline Standing up & $58.1 \%$ & $65.1 \%$ & $71.6 \%$ & $73.1 \%$ & $68.5 \%$ & $74.2 \%$ & $81.8 \%$ & $82.9 \%$ \\
\hline Overall & $69.8 \%$ & $87.1 \%$ & $89.8 \%$ & $94.3 \%$ & $84.6 \%$ & $90.9 \%$ & $93.9 \%$ & $96.6 \%$ \\
\hline
\end{tabular}

\section{Conclusion}

We investigated the impact of number, placement and type of the sensors on the accuracy of activity/posture recognition. We found that combination of different sensors achieved the desired accuracy in complex scenarios even when using a small number of sensors. Our goal was to recognize seven activities/postures. With one sensor placed on the chest, the classification model was able to distinguish between two groups of postures (\{lying and on all fours $\}$ and \{standing, sitting and sitting on the ground $\}$ ). This is because of the difference in the sensor angle orientation. The problems emerged while distinguishing postures within each group. That was the case even when the gyro and magnetometer data were analyzed. But in this case sitting was improved significantly (42 percentage points) because the system had better sensor orientation estimation.

When including the ankle right sensor in acceleration hardware scenario, the overall accuracy improved up to $87.1 \%$. The combination of chest and thigh sensor achieved accuracy of $89.8 \%$. The situation is similar in the other hardware scenario that includes gyro and magnetometer. This helps us to conclude that thigh is better placement for these 7 target activities/postures.

Three sensors improved the accuracy for 5 percentage points in acceleration scenario and almost 3 percentage points in the other scenario. Our experiments proved that using only accelerometers one can build good activity/posture recognition module. Including a gyroscope and magnetometer modules will improve the performances of the system.

The results of this study indicate that using only two sensors can give fairly good results in the accuracy of activity recognition process. The problem exists in sitting and sitting on the ground. This is the case because each person has its own way of sitting on the ground and it is difficult for the classification model to adapt to each person.

The comparison in accuracy using different sensor body placements is one of the original elements in this paper. This gives an overall insight about the best and most informative places on the body for the activity/posture recognition.

The first issue that needs to be addressed for the future work is developing more sophisticated methods for recognizing the transitional activities/postures (standing up, going down). This may make it possible to distinguish between standing and sitting with only one sensor.

Also, adding one more different type of sensor (e.g. location sensor), should improve the results and make the system more efficient. By using a position sensor the system will be aware of the location of the user (which room, which part of the room, is he/she laying on the bed or floor etc.).

Another aspect that could be improved is adding a detection of falls. The idea is to use independent module only for detecting falling patterns, because the whole process of falling down is very similar to the activity of fast going down.

\section{References}

[1] i2010 - A European Information Society for growth and employment website. [Accessed: February, 2011] http://ec.europa.eu/information_society/eeurope/i2010/ind ex_en.htm.

[2] V. Mirchevska, M. Luštrek, and M. Gams, Combining machine learning and expert knowledge for classifying human posture, Proc. of International Electrotechnical 
and Computer Science Conference 2009, pp. 183-186, 2009.

[3] M. Luštrek and B. Kaluža, Fall detection and activity recognition with machine learning, Proc. of Informatica 33(2): pp. 197-204, 2009.

[4] B. Kaluža, V. Mirchevska, E. Dovgan, M. Luštrek and M. Gams, An agent-based approach to care in independent living, Proc. of AmI 2010, pp. 177-186, 2010.

[5] Confidence project website. [Accessed: February, 2011]: http://www.confidence-eu.org.

[6] Ubisense RTLS website. [Accessed: February, 2011]: http://www.ubisense.net.

[7] N. Ravi, N. Dandekar, P. Mysore and M. L. Littman, Activity recognition from accelerometer data, Proc. IAAI'05 of the 17th conference on Innovative applications of artificial intelligence.

[8] H. Zhenyu, J. Lianwen, Activity recognition from acceleration data based on discrete consine transform and SVM, Proc. of the 2009 IEEE International Conference on Systems, Man, and Cybernetics.

[9] J. Yang, Y. Chen, G. Lee, S. Liou, and J. Wang, Activity Recognition Using One Triaxial Accelerometer: A Neurofuzzy Classifier with Feature Reduction, Proc. ICEC, 2007, pp.395-400.

[10] C.-F. Lai, Y.-M. Huang, H.-C. Chao, and J. H. Park, Adaptive Body Posture Analysis Using Collaborative Multi-Sensors for Elderly Falling Detection, IEEE Intelligent Systems (SCI), IEEE CS, Vol. 25, Iss. 2, pp. 2030, 2010.

[11] H.-T. Lin, Y.-J. Hsieh, M.-C. Chen, and W.-R. Chang, ActionView: A Movement-Analysis Ambulatory Monitor in Elderly Homecare Systems, in Proc. IEEE ISCAS'09, pp. 3098-3101, May 2009. (EI).

[12] Q. Li, J. A. Stankovic, M. Hanson, A. Barth, J. Lach, , and G. Zhou, Accurate, fast fall detection using gyroscopes and accelerometer-derived posture information. Proc. International Conference on Body Sensor Networks2009.

[13] XSens sensors website. [Accessed: February, 2011]: http://www.xsens.com/.

[14] L. Breiman, "Random Forests", Machine Learning, 45, pp. 5-32, 2001.

[15] Maths - Conversion Quaternion to Euler [Accessed: February, 2011]:

http://www.euclideanspace.com/maths/geometry/rotations /conversions/quaternionToEuler/index.htm. 